\title{
RESPUESTA DEL TOMATE DE CÁSCARA SILVESTRE MEXICANO (Physalis spp.) AL POTENCIAL OSMÓTICO EN HIDROPONÍA
}

\author{
RESPONSE OF MEXICAN WILD HUSK TOMATO (Physalis spp.) \\ TO OSMOTIC POTENTIAL IN HYDROPONICS
}

\author{
Roberto Chamú-Juárez, Manuel Sandoval-Villa*, Ma. de las Nieves \\ Rodríguez-Mendoza y Antonio García-Esteva
}

Colegio de Postgraduados, Campus Montecillo, Texcoco, Estado de México, México,

*Autor de correspondencia (msandoval@colpos.mx)

\section{RESUMEN}

El potencial osmótico $\left(\Psi_{0}\right)$ de las soluciones nutritivas puede influir en el crecimiento y la producción; sin embargo, el efecto está en función de la magnitud del $\Psi_{0}$ y de la demanda 0 capacidad de absorción de cada especie. El objetivo de la presente investigación fue determinar el crecimiento y rendmiento de tomate de cáscara silvestre mexicano (Physalis spp.) a diferentes potenciales osmóticos en la solución nutritiva. Se evaluaron los materiales $9 \mathrm{~b}, 17 \mathrm{~b}, 7 \mathrm{~b}$ y Filadelfia con $\psi_{0}$ de $-0.018,-0.036$ y $-0.054 \mathrm{MPa}$ bajo condiciones de invernadero e hidroponía. Se cuantificó el efecto de los tratamientos a los 30,60 y 90 días después del trasplante (ddt) a través de mediciones de altura de planta, diámetro de tallo, número de flores por planta y lecturas de la intensidad del color verde de las hojas, o lecturas SPAD (Soil Plant Analysis Development). El porcentaje de flores caídas se determinó a los $90 \mathrm{ddt}$, y durante un mes se cuantificó el porcentaje de amarre de frutos, número de frutos por planta y peso de los frutos. En general, todas las variables fueron afectadas por el $\Psi_{0}$ de la solución nutritiva y por el material vegetal. La altura de planta, diámetro de tallo, número de flores y las lecturas SPAD fueron mayores conforme aumentó la concentración de la solución nutritiva (menor $\Psi_{0}$ ); sin embargo, el porcentaje de flores caídas, amarre de frutos, número y peso de frutos fueon menores al exponer las plantas a-0.054 MPa. El material $9 \mathrm{~b}$ alcanzó mayor altura y el $17 \mathrm{~b}$ incrementó en mayor medida el diámetro con respecto a los otros materiales. Los genotipos $9 \mathrm{~b}$ y $17 \mathrm{~b}$ aumentaron el número de flores; $7 \mathrm{~b}$ y $17 \mathrm{~b}$ tuvieron el mayor porcentaje de flores caídas, $7 \mathrm{~b}$ presentó las mayores lecturas SPAD y la mayor producción se consiguió con el material $9 \mathrm{~b}$. Todos los genotipos presentaron poco crecimiento con -0.018 $\mathrm{MPa}$ en la solución nutritiva y con un potencial osmótico elevado se redujo el rendimiento de frutos. El nivel intermedio $(-0.036 \mathrm{MPa})$ es el potencial osmótico apropiado para la producción de frutos. La accesión $9 \mathrm{~b}$ fue la más adecuada para la producción bajo invernadero porque mostró el mayor número de frutos y rendimiento.

Palabras clave: Physalis spp., accesión, concentración nutrimental, material genético, solución nutritiva.

\section{SUMMARY}

The osmotic potential $\left(\Psi_{0}\right)$ of nutrient solutions can influence growth and production; however, the effect is a function of the magnitude of $\Psi_{0}$ and the demand or the absorption capacity of each species. The objective of this research was to evaluate growth and yield of Mexican wild husk tomato (Physalis spp.) at different osmotic potentials in the nutrient solution. Accessions $7 b, 9 b, 17 b$ and Philadelphia were evaluated with $\psi_{0}$ of -0.018 , -0.036 and $-0.054 \mathrm{MPa}$ under greenhouse conditions and hydroponics.
The effect of treatments at 30,60 and 90 days after transplant (DAT) was quantified through measurements of plant height, stem diameter, number of flowers per plant and readings for the intensity of the green color of leaves, or SPAD (Soil Plant Analysis Development) readings. The percentage of fallen flowers was determined at $90 \mathrm{DAT}$, and during one month the percentage of fruit set, number of fruits per plant and weight of fruits were quantified. In general, all traits were affected by the $\psi_{0}$ of the nutrient solution and by plant material. Plant height, stem diameter, number of flowers, and SPAD readings were higher as nutrient solution concentration increased (lower $\Psi_{0}$ ). However, the percentage of fallen flowers, fruit set, fruit number and fruit weight were lower when plants were exposed to $-0.054 \mathrm{MPa}$. Accession $9 \mathrm{~b}$ reached a larger height, and $17 \mathrm{~b}$ increased its diameter more than other materials. Accessions $9 \mathrm{~b}$ and $17 \mathrm{~b}$ increased the number of flowers; $7 \mathrm{~b}$ and $17 \mathrm{~b}$ registered the highest percentage of fallen flowers, $7 \mathrm{~b}$ had the highest SPAD readings, and the highest yield was achieved with accession $9 \mathrm{~b}$. All genotypes showed reduced growth with $-0.018 \mathrm{MPa}$ in the nutrient solution, and with high osmotic potential fruit yield decreased. The intermediate level $(0.036$ $\mathrm{MPa}$ ) is the appropriate osmotic potential for fruit production. Accession $9 \mathrm{~b}$ was the most suitable for fruit production under greenhouse conditions as it showed the highest number of fruits and yield.

Index words: Physalis spp., accession, genetic material, nutrient concentration, nutrient solution.

\section{INTRODUCCIÓN}

Physalis es un género americano de interés para ser cultivado, ya que varias especies proporcionan frutos comestibles (López-Sandoval et al., 2018a), por lo que diversos estudios se han enfocado a mejorar la producción (López-Sandoval et al., 2018b) y la calidad del fruto (Gastelum-Osorio et al., 2013; Peña-Lomelí et al., 2014); sin embargo, en la literatura no hay estudios relacionados con la respuesta del tomate silvestre mexicano al potencial osmótico $\left(\Psi_{0}\right)$ de la solución nutritiva en condiciones de invernadero que pudiera influir en una mayor producción que en el medio donde se desarrolla naturalmente.

El $\psi_{\text {o }}$ es consecuencia de los solutos disueltos, con característica intrínseca de disminuir la energía libre del agua, el cual se encuentra relacionado con la fracción molar 
del agua o con su actividad y es parte del componente del potencial hídrico (Sánchez-Díaz y Aguirreolea, 2008); además, es el constituyente del potencial del agua que resulta de la presencia de partículas de soluto, que es equivalente a la presión osmótica en concepto, pero de signo negativo. Urrieta-Velázquez et al. (2012), al evaluar tres selecciones de tomate de costilla (Solanum lycopersicum L.) expuestas a dos $\psi_{0}(-0.036$ y -0.072 $\mathrm{MPa}$ ) de la solución nutritiva Steiner, encontraron que las selecciones presentaron mejor comportamiento cuando fueron regadas a un $\psi_{\text {o de }}-0.072 \mathrm{MPa}$. Por su parte, Valentín-Miguel et al. (2013) obtuvieron en chile de agua (Capsicum annuum L.) rendimientos mayores de $2 \mathrm{~kg}$ de fruto comercial en plantas regadas con un $\psi_{0}$ menor a -0.054 MPa. Por otra parte, Rojas-Velázquez et al. (2013) evaluaron el $\psi_{0}$ de la solución nutritiva sobre Antirrhinum majus (L.) en dos estaciones del año; en invierno las plantas presentaron mejor respuesta cuando se empleó la solución con un $\psi_{0}$ de -0.072 y - $0.090 \mathrm{MPa}$ y en verano con un $\psi_{\text {o }}$ de $-0.036 \mathrm{MPa}$.

En este contexto, el objetivo de la presente investigación fue determinar la respuesta en el crecimiento y la producción de frutos de cuatro genotipos de tomate de cáscara silvestre mexicano (Physalis spp.) a diferentes potenciales osmóticos en la solución nutritiva.

\section{MATERIALES Y MÉTODOS}

\section{Sitio experimental}

La investigación se realizó en el Colegio de Postgraduados, Campus Montecillo, Estado de México, (19²9' 05" N, $98^{\circ}$ 54' 09" O, a 2240 m de altitud). Las plantas se cultivaron en un invernadero con techo de dos aguas, con cubierta de polietileno calibre 720, de transmitancia de luminosidad del $65 \%$. Las condiciones ambientales en el invernadero fueron humedad relativa al interior del invernadero de 10.1 a $96.0 \%$ (día y noche), temperatura promedio de $20.9^{\circ} \mathrm{C}$, máxima de $41.6^{\circ} \mathrm{C}$ y mínima de $8.4^{\circ} \mathrm{C}$. El estudio se realizó de mayo a septiembre de 2017.

\section{Material genético}

Se utilizaron cuatro genotipos de tomate de cáscara silvestre mexicano (Physalis spp.): las accesiones 7b (167 BHGEN), 9b (170 BNGEN) y 17b (48 NNGEN) provenientes del Banco Nacional de Germoplasma de Especies Nativas de la Universidad Autónoma Chapingo, donadas por el Dr. Aureliano Peña Lomelí, así como el material Filadelfia, el cual fue colectado en el municipio de Tlapehuala, región de Tierra Caliente, Guerrero, por el M.C. Roberto Chamú Juárez.

\section{Manejo agronómico}

Previo a la siembra, las semillas se escarificaron mediante raspado con una lija de agua de grano 80 de carburo de silicio y $230 \times 1280$ mm; además, se remojaron en agua destilada dentro de cajas de Petri a $25^{\circ} \mathrm{C}$ por $24 \mathrm{~h}$, con el objeto de promover una germinación uniforme. La siembra se llevó a cabo el 12 de abril de 2017 en charolas de poliuretano de 200 cavidades rellenas con turba (peat moss) húmeda. Se depositaron dos semillas por cavidad. A los 5 días después de la siembra (dds) los semilleros se extendieron sobre bancales dentro de un invernadero de cristal. Desde la siembra hasta que las plántulas presentaron la primera hoja verdadera se regaron con agua de la llave; posteriormente, a los 20 dds el riego se llevó a cabo alternadamente con agua y solución nutritiva Steiner (1984) al $25 \%$, a los 25 dds se aumentó al 50 \% y a los 30 dds al $75 \%$ de su fuerza iónica.

El trasplante se realizó el día 16 de mayo de 2017 (35 dds). Los recipientes fueron bolsas de polietileno de color negro de $30 \times 30 \mathrm{~cm}$ con capacidad de $13 \mathrm{~L}$, y como sustrato se utilizó tezontle rojo, con una granulometría de diámetro promedio de $2.17 \mathrm{~mm}$, índice de grosor de $71 \%$, densidad aparente promedio de $1.1 \mathrm{~g} \mathrm{~cm}^{-3}, 51 \%$ de porosidad total, $13 \%$ de porosidad de aireación, 38 $\%$ de retención de humedad y $13 \%$ de agua fácilmente disponible. Se utilizó una densidad de población de 11 plantas por $\mathrm{m}^{2}$ (90 cm entre surcos y $20 \mathrm{~cm}$ entre plantas) con doble hilera de bolsas. Previo al trasplante, se aplicó un tratamiento preventivo con una mezcla de Previcur ${ }^{\circledR}$ $72.2 \%$ (Propamocarb HCL) en dosis de $0.5 \mathrm{~mL} \mathrm{~L}^{-1}$ para prevenir hongos y de Confidor ${ }^{\circledR} 350$ SC (Imidacloprid) 1 $\mathrm{mL} \mathrm{L-1}$ contra plagas.

Las soluciones nutritivas se prepararon con base en la solución nutritiva Steiner completa $\left(12 \mathrm{NO}_{3}-1 \mathrm{H}_{2} \mathrm{PO}_{4}^{-}, 7\right.$ $\mathrm{SO}_{4}{ }^{2-}, 7 \mathrm{~K}^{+}, 9 \mathrm{Ca}^{2+}$ y $4 \mathrm{Mg}^{2+}$ meq $\left.\mathrm{L}^{-1}\right)$ con un $\Psi_{0}$ de -0.072 $\mathrm{MPa}$, complementada con micronutrimentos $\mathrm{Fe}, \mathrm{B}, \mathrm{Mn}$, Zn, Cu y Mo con 3, 0.5, 0.7, 0.09, 0.02 y $0.04\left(\mathrm{mg} \mathrm{L}^{-1}\right)$. La conductividad eléctrica (CE) de las soluciones fue 0.5, 1.0 y $1.5 \mathrm{dS} \mathrm{m} \mathrm{m}^{-1}$. El pH se manejó a un intervalo de 5.5 a 6.5 y se usó ácido sulfúrico $1 \mathrm{~N}$ para ajustarlo cada tercer día, medido con un potenciómetro PC18 (Conductronic, Puebla, México).

Las plantas se regaron por goteo, con goteros cuyo gasto fue de $8 \mathrm{~L} \mathrm{~h}^{-1}$. Después del trasplante se aplicaron tres riegos de 1 min de duración por día, a los 15 d se aumentó a nueve riegos por día con una duración de 3 min en la etapa vegetativa, 5 min en la de floración y 7 min en la etapa de fructificación. Se colocaron tutores de rafia tomatera a los 8 días después del trasplante (ddt). El sistema de riego se lavó cada 15 días con agua acidulada a 
pH 4.5 para evitar la saturación de sales en los goteros y en el sustrato. Considerando la susceptibilidad que presenta el género Physalis contra la cenicilla (Oidiopsis taurica), se realizaron aplicaciones preventivas con Precure ${ }^{\circledR}$ (Triflumizole) a una dosis de $2 \mathrm{~mL} \mathrm{~L}^{-1}$ cada $15 \mathrm{~d}$.

Para prevenir la incidencia de mosquita blanca (Trialeurodes vaporariorum) se aplicaron $2 \mathrm{~mL} \mathrm{~L}^{-1}$ de Oberon® SC 240 (Spiromesifen) cada 15 d y para evitar daños por gusano del fruto (Heliothis subflexa) se realizaron aplicaciones de Karate Zeon ${ }^{\circledR} 5$ CS (Lambda cyalotrina) a dosis de $3 \mathrm{~mL} \mathrm{~L}^{-1}$ cada 15 días después del cuajado del fruto.

\section{Tratamientos y diseño experimental}

La combinación de los cuatro genotipos (9b, 17, $7 \mathrm{~b}$ y Filadelfia) y tres niveles de $\Psi_{0}(-0.018,-0.036$ y $-0.054 \mathrm{MPa})$ generaron 12 tratamientos, valores que se derivaron de la ecuación de van't Hoff: $\Psi_{0}=-$ RTCs; donde $R$ es la constante de los gases $8.32 \mathrm{~J} \mathrm{~mol}^{-1} \mathrm{~K}^{-1}$, T es la temperatura absoluta en grados Kelvin y Cs es la concentración molar de soluto en solución (Taiz y Zieger, 2002). Los tratamientos se distribuyeron en un diseño experimental completamente al azar con cuatro repeticiones y con arreglo en parcelas divididas; en la parcela grande se ubicaron los niveles de potencial osmótico y en la parcela chica los genotipos. La unidad experimental consistió en una planta colocada en una bolsa negra de polietileno con capacidad de $13 \mathrm{~L}$.

\section{Variables evaluadas}

Las variables evaluadas a los 30,60 y 90 ddt fueron altura de planta en $\mathrm{cm}$, medida con un flexómetro desde la base del tallo (cuello de la planta) hasta el ápice de la rama más larga; diámetro de tallo en mm, con un vernier digital marca Truper $\AA$ a $5 \mathrm{~cm}$ del cuello de la planta; número de flores totales por planta y lecturas SPAD con el equipo SPAD-502 (Konica Minolta, Osaka, Japón) en cuatro hojas recientemente expandidas ubicadas hacia los cuatro puntos cardinales de la planta en horario lo más próximo posible al medio día.

El porcentaje de flores caídas se calculó mediante la diferencia entre el número de flores y la acumulación de frutos cosechados a los $90 \mathrm{ddt}$, ya que en estos materiales no hay caída de frutos. Para evaluar el rendimiento, se cosechó y cuantificó el número total de frutos maduros producidos por planta, se registró su peso en g incluyendo el cáliz con una balanza electrónica de laboratorio portátil Ohaus ${ }^{\circledR}$ (Ohaus Corp., Parsippany, Nueva Jersey, EUA) y al final se obtuvo la suma del número y el peso de los frutos.

\section{Análisis estadístico}

A las variables en estudio se les aplicó análisis de varianza (ANDEVA) y prueba de comparación medias de Tukey $(P \leq 0.05)$ mediante el programa estadístico SAS, versión 9.1 (SAS Institute, 2011).

Debido a que no se cumplió el supuesto de normalidad, y considerando el efecto significativo del análisis de varianza en las variables relacionadas con el rendimiento (número y peso de frutos), a los datos se les aplicó una transformación logarítmica con el objeto de ajustarse al cumplimiento de dichos supuestos. La representación gráfica de los valores se realizó con el programa SigmaPlot de Jandel Scientific versión 13, para comparar el efecto del potencial osmótico y los genotipos.

\section{RESULTADOS Y DISCUSIÓN}

En general, el $\Psi_{\text {o }}$ influyó sobre las variables morfológicas y sobre el rendimiento. La accesión $9 b$ fue la más promisorio para rendimiento en hidroponía e invernadero. Se encontró interacción significativa entre los factores principales (genotipos y potenciales osmóticos) a los 90 ddt, por lo que sólo la información de esa fecha se presenta y discute para dicha interacción.

\section{Efecto del potencial osmótico sobre el crecimiento}

El ANDEVA mostró diferencias significativas $(P \leq 0.05)$ entre $\Psi_{\text {o }}$ para altura de planta y diámetro de tallo (Figura 1). La altura de planta se afectó sólo a los 30 ddt (Figura 1A), mientras que el diámetro de tallo en todas las fechas de muestreo (Figura 1B). A los 30 ddt, las plantas más altas fueron aquellas con los $\psi_{0}$ de -0.054 y $-0.036 \mathrm{MPa}$ (55.90 cm y $51.68 \mathrm{~cm}$, respectivamente), mientras que en los muestreos de 60 y $90 \mathrm{ddt}$ las diferencias no fueron significativas. Con respecto al diámetro, a mayor potencial mayor fue la expresión del diámetro, aunque sólo a los 30 ddt las diferencias fueron significativas. A los 60 y $90 \mathrm{ddt}$ sólo se diferencian los tallos de la menor concentración en la solución nutritiva con respecto a la mayor.

Cruz et al. (2012) determinaron que la altura de planta y el diámetro de tallo de tomate Charleston se relaciona directamente con el aumento en la concentración de la solución nutritiva. El efecto en la altura se atribuye a la mayor absorción nutrimental (Miranda-Villagómez et al., 2014); sin embargo, con el paso del tiempo este comportamiento tiende a presentar una respuesta inversa, lo cual se atribuye a la acumulación de sales en el sustrato (Bustomi Rosadi et al., 2014). Cruz-Crespo et 
al. (2014) obtuvieron tallos más gruesos en chile serrano (Capsicum annuum) con 75 \% (-0.054 MPa) de la solución nutritiva Steiner, con respecto a $25 \%$ (-0.018 MPa), valores similares a los encontrados en la presente investigación, mientras que Miranda-Villagómez et al. (2014) obtuvieron mejores promedios con el $50 \%\left(\Psi_{0}=-0.036 \mathrm{MPa}\right)$ en tallos florales de Freesia $\times$ hybrida, por lo que este trabajo sugiere que la respuesta depende de la especie. Finalmente, se observó que un alto $\psi$ tiene efecto negativo sobre la altura de la planta a lo largo del tiempo, lo cual se debe a la acumulación de sales en el sustrato y a que el diámetro basal es menos afectado por el $\Psi_{0}$.

\section{Crecimiento de los genotipos}

Se observaron diferencias significativos $(P \leq 0.05)$ a los 30 y 60 ddt entre los genotipos en altura de planta (Figura

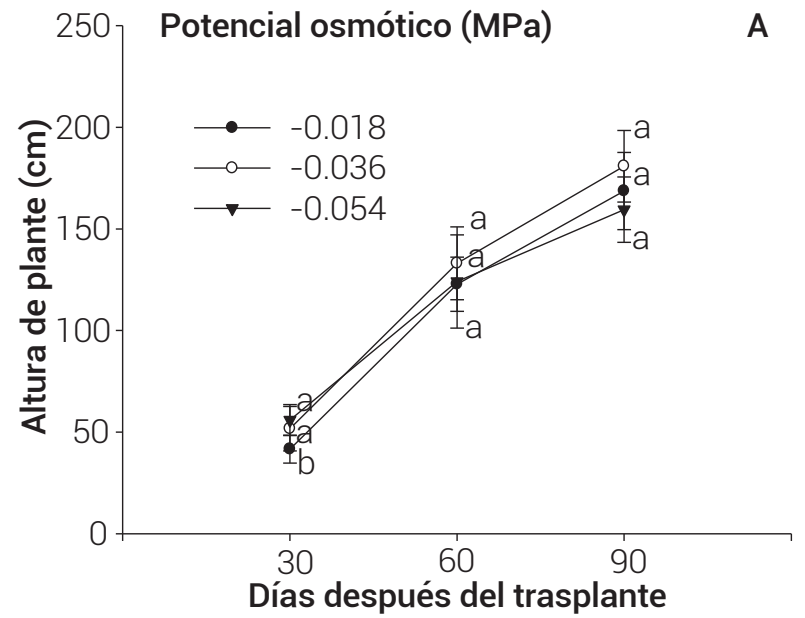

2A) y en diámetro de tallo (Figura 2B). A los 30 y 60 ddt los materiales $9 \mathrm{~b}, 17 \mathrm{~b}$ y $7 \mathrm{~b}$ presentaron mayor altura de planta, superando a Filadelfia. A los 30 ddt 17b sobresalió en el diámetro de tallo con $12.4 \mathrm{~mm}$. A los 60 ddt no hubo diferencias significativas y a los 90 ddt $9 b$ fue el de menor diámetro; es decir, el genotipo más alto fue el más delgado. Para producción en invernadero es de interés que las plantas sean pequeñas y de tallos gruesos; por lo tanto, por el diámetro, los materiales 17b y $7 \mathrm{~b}$ serían los mejores, dado que a los $90 \mathrm{ddt}$ todos los materiales tuvieron la misma altura.

Peña-Lomelí et al. (2014) indicaron que la expresión del potencial de los cultivos está relacionada directamente con el genotipo, el ambiente y la interacción genotipo $x$ ambiente, por lo que la respuesta de una mayor altura y diámetro de tallo del material 9b a los 30 y 60 ddt puede

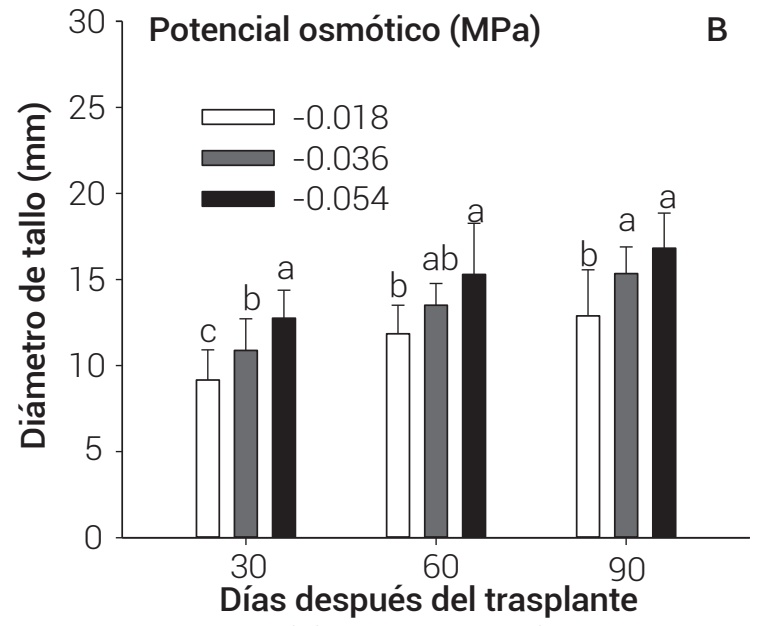

Figura 1. Efecto del potencial osmótico $\left(\Psi_{\mathrm{o}}\right)$ sobre la altura $(A)$ y el diámetro de tallo $(B)$. Medias $\pm \mathrm{DE}$ (desviación estándar) con las mismas letras entre $\Psi_{\circ}$ en cada muestreo son estadísticamente iguales (Tukey, $\mathrm{P} \leq 0.05$ ).
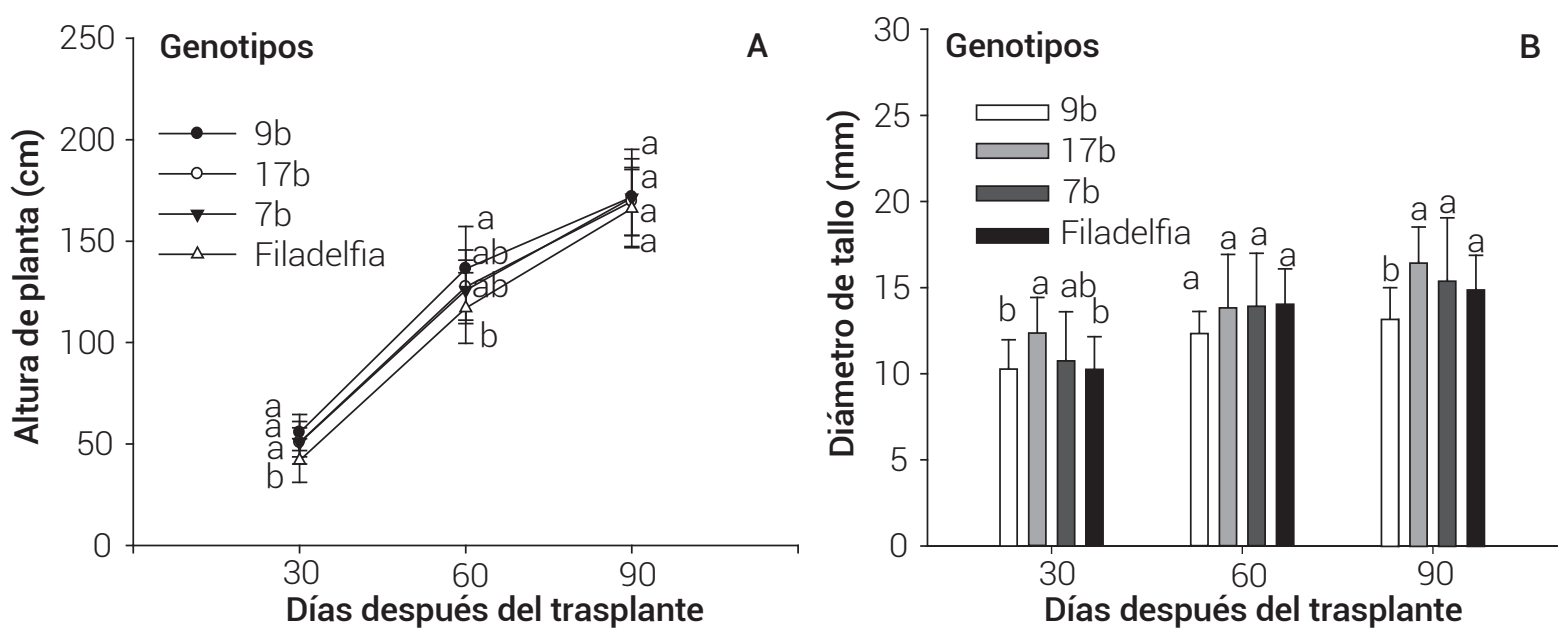

Figura 2. Altura de planta (A) y el diámetro de tallo (B) de genotipos de tomate de cáscara mexicano (Physalis spp.). Medias $\pm D E$ (desviación estándar) con letras iguales entre los genotipos en cada muestreo son estadísticamente iguales (Tukey, $\mathrm{P} \leq 0.05$ ). 
ser característica genética o una respuesta favorable a la interacción genotipo $\times$ ambiente. El mayor diámetro de tallo a los 30 y 90 ddt correspondió a los materiales $17 \mathrm{~b}$ y $7 \mathrm{~b}$. Posiblemente, el bajo nivel de producción de estos materiales les permitió aumentar su diámetro de tallo; sin embargo, para el caso de los materiales 9b y Filadelfia, es probable que presenten una alta capacidad de absorción nutrimental, lo que les permitió asignar sus reservas a sitios con mayor demanda y así aumentar la producción de frutos.

\section{Interacción potencial osmótico $\times$ genotipo}

El análisis estadístico mostró interacción significativa entre los $\psi_{0}$ y los materiales genéticos a los $90 \mathrm{ddt}$ (Cuadro 1). El genotipo 7b presentó el mayor diámetro de tallo cuando el $\psi_{0}$ de la solución nutritiva fue de -0.054 $\mathrm{MPa}$. Dicho incremento en el diámetro fue de $13.79 \%$ en comparación con las plantas que se regaron con la solución cuyo $\psi_{\text {。 }}$ fue de $-0.036 \mathrm{MPa}$. Por otra parte, los resultados indicaron que solamente el material $7 \mathrm{~b}$ fue capaz de tolerar altas concentraciones de la solución $\left(\right.$ menor $\psi_{0}$ ) y la cantidad de nutrimentos suministrados en la solución no satisfizo la demanda nutrimental de los materiales 9b, 17b y Filadelfia. Martínez et al. (2008) mencionaron que la nutrición modifica las características morfológicas y el crecimiento, pero si la concentración de un elemento está por debajo de lo requerido, se afecta el desarrollo y provoca alteraciones en los procesos metabólicos; por el contrario, si este efecto no fue causado por la alta demanda nutrimental, sino por el exceso de sales en el sustrato, las plantas presentan estrés. Goykovic y Saavedra (2007) señalaron que la alta concentración de sales puede provocar alteraciones en los órganos del dosel vegetal; además, con tallos de menor altura se reduce el número de hojas y se presenta desecación en los bordes, de modo que hay menos síntesis de fotoasimilados.

\section{Influencia del potencial osmótico sobre el número de flores y porcentaje de flores caídas}

A los $30 \mathrm{ddt}$, el mayor número de flores se obtuvo con el $\psi$ de $-0.054 \mathrm{MPa}$ (17.93 flores por planta, Figura 3A, P $\leq 0.05$ ), una diferencia de $19.53 \%$ respecto a la solución con $\psi_{0}$ de -0.018 MPa. Miranda-Villagómez et al. (2014) reportaron resultados similares en crecimiento y calidad de tallo floral de Freesia $\times$ hybrida en hidroponía, observaron mayor número de flores al incrementar la concentración de la solución nutritiva (menor $\psi_{\text {o }}$ ). Bustomi Rosadi et al. (2014) expusieron plantas de Solanum lycopersicum a diferentes conductividades eléctricas (CE) y encontraron que éstas aumentaron el número de flores al incrementar la CE; sin embargo, con concentraciones muy altas se obtuvo un resultado contrario porque la salinidad deteriora el proceso de absorción de agua y nutrimentos. Los resultados en esta investigación sugieren que las plantas con baja CE sufrieron deficiencias nutrimentales, pero cuando la CE fue alta las plantas sufrieron por salinidad.

\begin{tabular}{lcc}
$\begin{array}{l}\text { Cuadro 1. Interacción entre el potencial osmótico }\left(\boldsymbol{\Psi}_{\mathrm{o}}\right) \text { de la solución nutritiva y los materiales } \\
\text { genéticos de tomate de cáscara mexicano }\end{array}$ \\
$\begin{array}{l}\text { (Physalis spp.) sobre el diámetro del tallo a los } 90 \text { días } \\
\text { después del trasplante. }\end{array}$ & Genotipo & Diámetro de tallo $(\mathrm{mm})$ \\
\hline$\Psi_{0}(\mathrm{MPa})$ & $7 \mathrm{~b}$ & $18.79 \pm 0.64 \mathrm{a}$ \\
\hline-0.054 & $17 \mathrm{~b}$ & $18.18 \pm 1.95 \mathrm{ab}$ \\
-0.054 & $7 \mathrm{~b}$ & $16.20 \pm 0.65 \mathrm{abc}$ \\
-0.036 & Filadelfia & $16.14 \pm 1.68 \mathrm{abc}$ \\
-0.036 & $17 \mathrm{~b}$ & $15.78 \pm 0.59 \mathrm{abc}$ \\
-0.036 & $17 \mathrm{~b}$ & $15.50 \pm 2.28 \mathrm{abc}$ \\
-0.018 & Filadelfia & $15.47 \pm 1.04 \mathrm{abc}$ \\
-0.054 & $9 \mathrm{~b}$ & $14.84 \pm 0.46 \mathrm{bcd}$ \\
-0.054 & $9 \mathrm{~b}$ & $13.30 \pm 0.91 \mathrm{~cd}$ \\
-0.036 & Filadelfia & $13.18 \pm 1.92 \mathrm{~cd}$ \\
-0.018 & $9 \mathrm{~b}$ & $11.49 \pm 1.81 \mathrm{~d}$ \\
-0.018 & $7 \mathrm{~b}$ & $11.33 \pm 3.01 \mathrm{~d}$ \\
-0.018 &
\end{tabular}

Medias \pm DE (desviación estándar) con letras iguales no son estadísticamente diferentes (Tukey P $\leq 0.05)$. 
En cuanto al porcentaje de flores caídas registradas al final del experimento, hubo diferencias significativas por efecto del $\Psi_{0}$ (Figura 3B). El mayor porcentaje se obtuvo bajo $\psi_{\text {o de }}-0.054$ y $-0.018 \mathrm{MPa}$, superando estadísticamente al $\psi$ intermedio, lo cual se explica por deficiencia nutrimental en el bajo suministro y por salinidad en el suministro elevado. Marcelis et al. (2004) reportaron que el número de flores caídas y frutos en chile dulce no sólo depende de la fuerza de la fuente sino también de la demanda de órganos competidores, ya que la fuerza total de la demanda es alta debido a que muchos frutos crecen simultáneamente. Por su parte, Wubs et al. (2009) indicaron que la fuerza de la demanda es más importante que la de la oferta, por lo que las flores y los frutos jóvenes no deberían competir por los fotoasimilados con los frutos de crecimiento rápido. Por lo anterior, una planta no debe ser de gran tamaño, sino abastecer la demanda que generen los órganos reproductores.

\section{Efecto del material genético sobre el número y el porcentaje de flores caídas}

Se observaron diferencias entre genotipos sobre el número de flores en todas las fechas de muestreo (Figura $4 \mathrm{~A})$; asimismo, los genotipos influyeron sobre el porcentaje de flores caídas (Figura 4B). A los 30 ddt, la mayor cantidad de flores correspondió al material 9b (24.00), y a los 60 y $90 \mathrm{ddt}$, al 9b (124.66 y 124.33) y al $17 \mathrm{~b}(280.00$ y 283.41$)$. Se encontró que los materiales $17 \mathrm{~b}$ y $7 \mathrm{~b}$ fueron los que presentaron el mayor porcentaje de flores caídas (86.94 y $87.28 \%$ ), que se obtuvo de la diferencia entre el número total de flores y el de frutos cosechados a los $90 \mathrm{ddt}$.

El material 9b presentó el mayor número de flores en los tres muestreos y Filadelfia presentó la menor cantidad; asimismo, en el porcentaje de flores caídas, $9 b$ fue el que menos flores perdió, seguido por Filadelfia, materiales que también produjeron la mayor cantidad de frutos, contrario a lo que sucedió en $17 \mathrm{~b}$ y $7 \mathrm{~b}$, que presentaron el mayor porcentaje de flores caídas, así como el menor número de frutos por planta. Esta respuesta pudo ser meramente fisiológica, propia de los materiales silvestres, o también porque las plantas crecieron libremente al no podarse, ya que la poda influye en el número de flores y en la calidad de frutos; sin embargo, cuando no se realiza, el crecimiento rápido de algún órgano compite con las hojas por nutrimentos, que fácilmente se pueden movilizar, provocando senescencia foliar y reducción en la fotosíntesis (Ponce et al., 2012).

\section{Efecto del potencial osmótico y los genotipos sobre las lecturas SPAD}

Las lecturas SPAD son un indicador indirecto del estatus nutrimental de la planta. Este índice mide la intensidad del color verde de las hojas. Un mayor valor de la lectura indica una nutrición cercana al óptimo, al menos para nutrimentos que influyen sobre la producción de clorofila. El $\psi_{\text {o }}$ tuvo efecto significativo sobre las lecturas SPAD en las tres fechas de muestreo (Figura 5A), y el material genético mostró diferencias sólo a los 60 y 90 ddt (Figura 5B). Los valores expresados por efecto del $\psi_{0}$ presentaron la misma tendencia en las tres fechas de muestreo, en las que se obtuvieron las lecturas más altas con los $\psi_{0}$ de -0.036 y -0.054 MPa. A los 60 ddt, las lecturas más altas se registraron en los materiales $9 \mathrm{~b}$ y $7 \mathrm{~b}$ y a los $90 \mathrm{ddt}$ solamente en $7 \mathrm{~b}$.
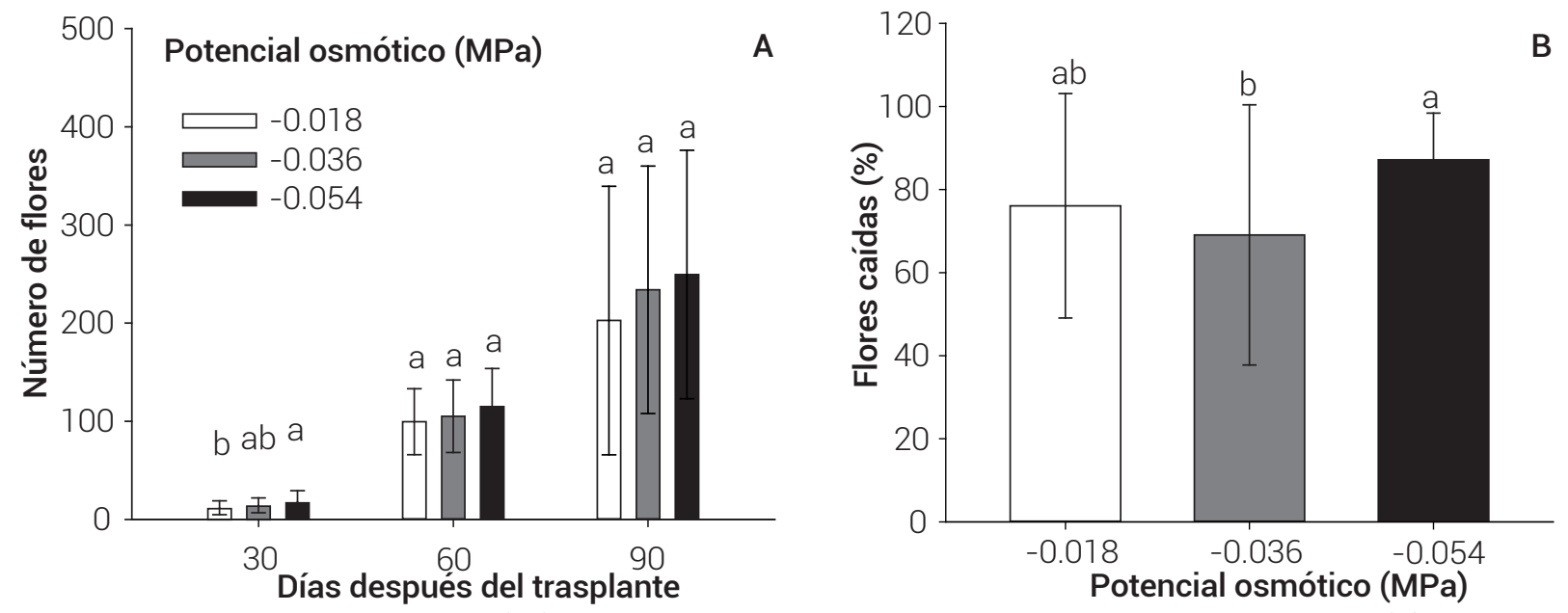

Figura 3. Efecto del potencial osmótico $\left(\Psi_{\mathrm{o}}\right)$ sobre el número de flores por planta a los 30,60 y $90 \mathrm{ddt}(\mathrm{A})$ y porcentaje de flores caídas a los 90 días después del trasplante (B). Medias \pm DE (desviación estándar) con letras iguales entre $\Psi_{\circ}$ en cada muestreo son estadísticamente iguales entre sí (Tukey, $\mathrm{P} \leq 0.05$ ). 

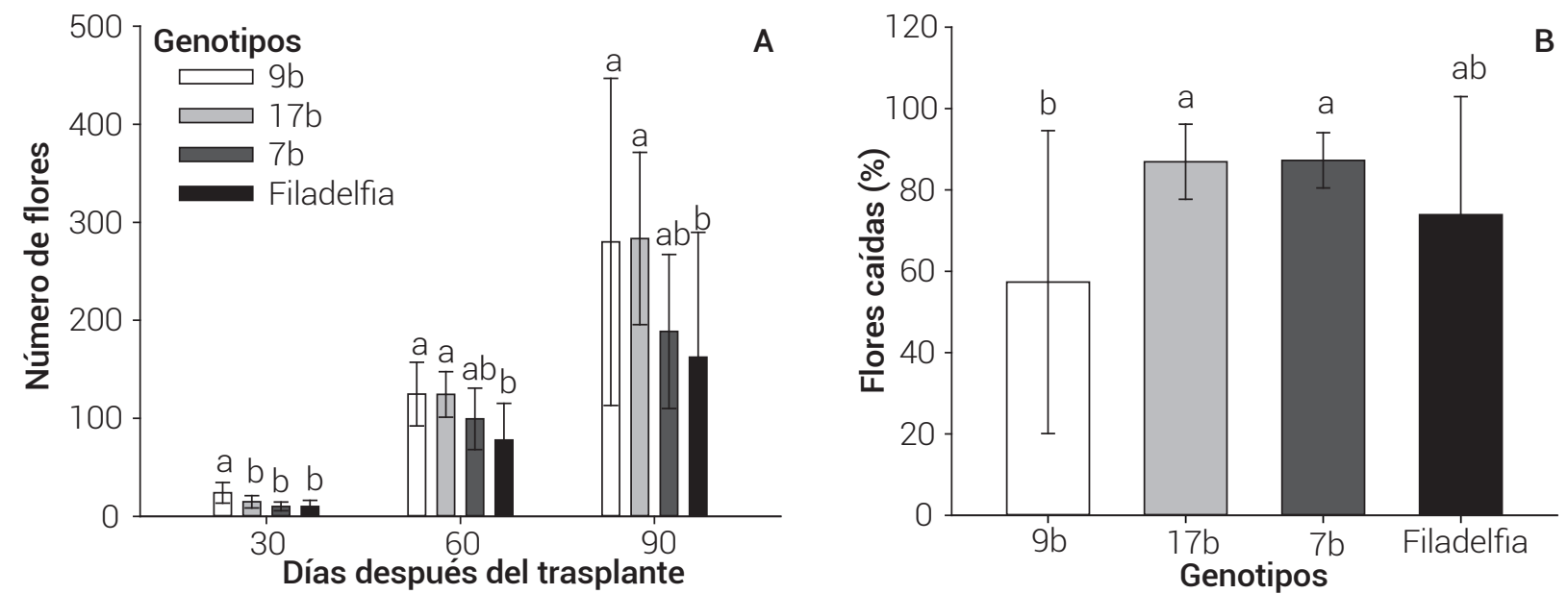

Figura 4. Efecto de los genotipos de tomate de cáscara silvestre mexicano sobre el número de flores a los 30,60 y 90 días después del trasplante (A) y porcentaje de flores caídas (B). Medias $\pm \mathrm{DE}$ (desviación estándar) con letras iguales entre genotipos en cada muestreo son estadísticamente iguales entre sí (Tukey, $\mathrm{P} \leq 0.05$ ).
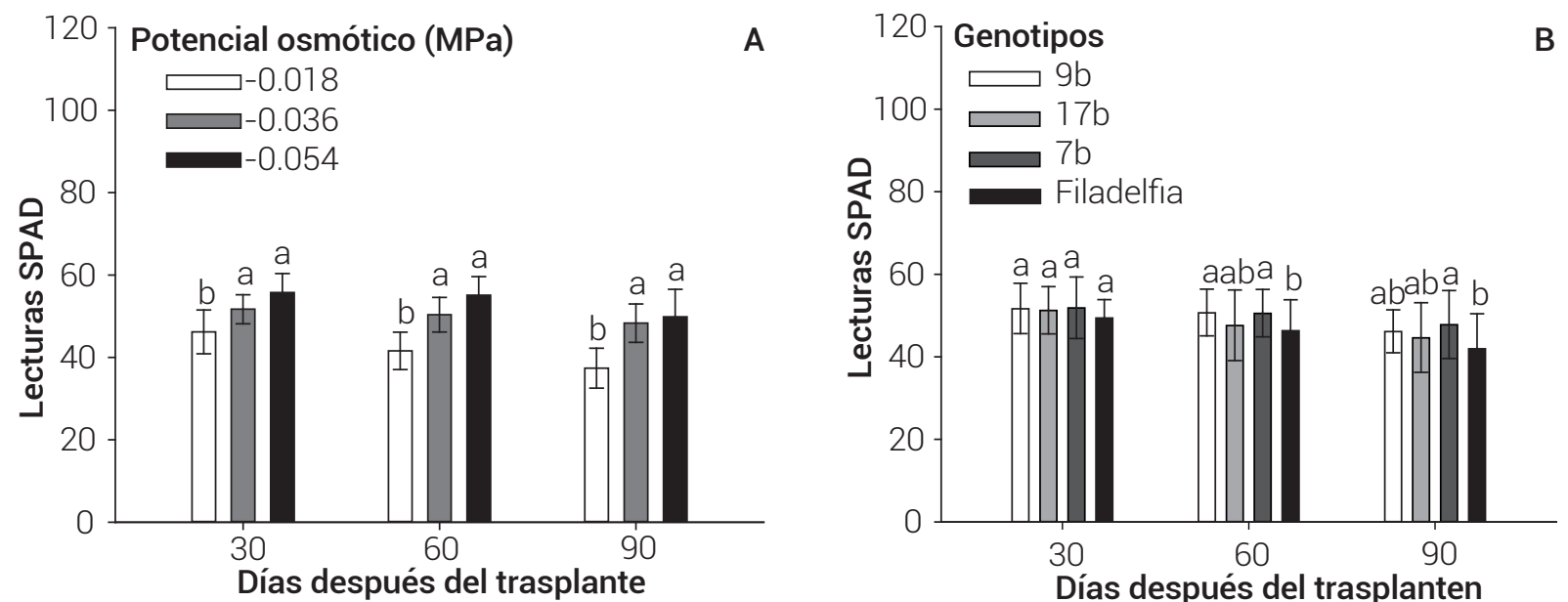

Figura 5. Efecto del potencial osmótico $\left(\Psi_{\circ}\right)(A)$ y de los genotipos $(B)$ sobre las lecturas SPAD en plantas de tomate de cáscara silvestre mexicano a 30,60 y 90 días después del trasplante. Medias $\pm D E$ (desviación estándar) con letras iguales entre $\Psi_{\circ}$ en cada muestreo son estadísticamente iguales entre sí (Tukey, $\mathrm{P} \leq 0.05$ ).

Las lecturas SPAD se incrementaron con la mayor concentración de la solución nutritiva (menor $\psi_{\text {o }}$ ), lo cual implica que en el suministro alto de nutrimentos hay mayor absorción de éstos. Resultados similares encontraron Cruz et al. (2012). Valenzuela et al. (2014), al incrementar la concentración de la solución nutritiva Steiner en plantas de tomate, observaron que aquellas expuestas a mayor concentración aumentaron este índice; sin embargo, en esta investigación se encontró que las lecturas SPAD disminuyeron con el paso del tiempo, lo cual coincide con el incremento de la demanda por el crecimiento de los frutos, y también se atribuye a la senescencia de las hojas, dado que los materiales son anuales. Según Ribeiro et al. (2015), los valores SPAD son más altos en plantas en desarrollo vegetativo; sin embargo, después de esta etapa las lecturas disminuyen, lo que coincide con la floración y fructificación; es decir, la planta moviliza nitrógeno de las primeras hojas hacia los frutos.

\section{Efecto del potencial osmótico sobre el amarre y producción de frutos}

Hubo efecto significativo del $\psi_{\text {o }}$ sobre el porcentaje de amarre, número y peso de frutos con cáliz (Cuadro 2). El irrigar las plantas con la solución nutritiva con $\psi$ 。 de $-0.036 \mathrm{MPa}$ aumentó el amarre de frutos en 58.54 $\%$, el número de frutos en $63.44 \%$ y el peso de frutos en $74.59 \%$ en comparación con el $\psi_{\text {。 }}$ de $-0.054 \mathrm{MPa}$. 
Cuadro 2. Efecto del potencial osmótico $\left(\Psi_{\mathrm{o}}\right)$ sobre el porcentaje de amarre de frutos, número y peso de frutos con cáliz acumulados al final del experimento.

\begin{tabular}{lccc}
\hline$\Psi_{0}(\mathrm{MPa})$ & Amarre de frutos (\%) & Número de frutos por planta & $\begin{array}{c}\text { Peso de frutos } \\
\text { (g por planta) }\end{array}$ \\
\hline-0.018 & $17.85 \pm 16.76 \mathrm{~b}$ & $54.33 \pm 65.03 \mathrm{ab}$ & $73.85 \pm 145.34 \mathrm{ab}$ \\
-0.036 & $30.92 \pm 31.32 \mathrm{a}$ & $74.68 \pm 83.20 \mathrm{a}$ & $110.29 \pm 156.58 \mathrm{a}$ \\
-0.054 & $12.82 \pm 11.24 \mathrm{~b}$ & $27.31 \pm 27.73 \mathrm{~b}$ & $28.03 \pm 33.51 \mathrm{~b}$ \\
$\mathrm{DSH}(0.05)$ & 11.56 & 51.69 & 122.29 \\
\hline
\end{tabular}

Medias \pm DE (desviación estándar) con letras iguales en las columnas no son estadísticamente diferentes (Tukey $\leq 0.05)$. DSH: diferencia

Con $\psi_{0}-0.018 \mathrm{MPa}$ se incrementó el amarre de frutos en $28.18 \%$, el número de frutos en $49.74 \%$ y el peso de frutos en $62.05 \%$ en comparación con el $\psi_{0}-0.054 \mathrm{MPa}$. Valentín-Miguel et al. (2013), al evaluar varios potenciales osmóticos de la solución nutritiva sobre el cultivo de chile de agua, obtuvieron rendimientos mayores de $2 \mathrm{~kg}$ de fruto comercial por planta con un $\psi_{0}$ menor de -0.054 $\mathrm{MPa}$; por lo anterior, no se requieren altas concentraciones en la solución nutritiva, pero sí que éstas contengan los nutrimentos necesarios para la producción de frutos.

En la presente investigación se encontraron menores rendimientos con la mayor concentración de nutrimentos en la solución nutritiva (-0.054 MPa), lo cual implica que los genotipos de tomate de cáscara son susceptibles a la salinidad. En hidroponía las plantas padecen por exceso de sales porque éstas acumulan sustrato con el paso del tiempo, lo que se refleja en una reducción en el número de flores (Ruiz et al., 2014); además, se incrementa la esterilidad y se altera la duración de la floración, lo que a su vez afecta la maduración de los frutos. Para estos materiales, niveles más altos de suministro nutrimental (-0.054 MPa) disminuyen la producción de frutos; contario a cuando se trabaja con -0.036 y $-0.018 \mathrm{MPa}$, donde hay mayor producción de frutos. Este efecto se atribuye a la naturaleza arvense de estos genotipos, adaptados a suelos con baja fertilidad y en competencia con otras especies de mayor interés agronómico en la milpa como maíz (Zea mays L.) y frijol (Phaseolus vulgaris).

\section{Efecto del material genético sobre el amarre y la producción de frutos}

Hubo efecto significativo del material sobre el porcentaje de amarre, número y peso de frutos con cáliz, siendo mayor en el material 9b para las tres variables (Cuadro $3)$. En todas las variables evaluadas, los valores más bajos correspondieron al material genético $7 \mathrm{~b}$. Barrera (2018, Com. Pers.) ${ }^{1}$, observó que el porcentaje de amarre con polinización manual fue de $67 \%$ en el material $7 \mathrm{~b}$, lo cual contrasta con los resultados del presente estudio, donde 9b presentó el mayor porcentaje de amarre (42 $\%)$ en forma natural en invernadero. Castro-Brindis et al. (2004) argumentaron que la expresión del potencial sobre el rendimiento de los cultivos está determinada por su constitución genética, el clima, las características del suelo, las condiciones nutrimentales, la técnica de producción y los factores biológicos; asimismo, por la alta actividad fotosintética y por la síntesis de carbohidratos. En general, el bajo número y peso de frutos de los materiales estudiados, en especial de $17 \mathrm{~b}$ y $7 \mathrm{~b}$, puede estar asociada a la autoincompatibilidad de los materiales, además de problemas con el aborto de frutos.

Para futuras investigaciones con estos materiales se sugiere comparar las mismas variables en estudios de

'Barrera I. C. A. (2018) Eficiencia de cruzas e identificación taxonómica de tres accesiones de tomate de cáscara (Physalis spp.). Tesis profesional. Departamento de Fitotecnia, Universidad Autónoma Chapingo. Chapingo, Estado de México. 32 p.

Cuadro 3. Efecto del material de Physalis spp. sobre el porcentaje de amarre de frutos, número y peso de frutos con cáliz acumulados al final del experimento.

\begin{tabular}{lccc}
\hline Genotipos & Amarre de frutos (\%) & Número de frutos por planta & Peso de frutos (g por planta) \\
\hline $9 \mathrm{~b}$ & $42.64 \pm 37.24 \mathrm{a}$ & $110.54 \pm 96.88 \mathrm{a}$ & $158.38 \pm 164.18 \mathrm{a}$ \\
$17 \mathrm{~b}$ & $13.05 \pm 9.22 \mathrm{~b}$ & $33.91 \pm 23.03 \mathrm{~b}$ & $28.15 \pm 18.73 \mathrm{~b}$ \\
$7 \mathrm{~b}$ & $12.71 \pm 6.79 \mathrm{~b}$ & $20.91 \pm 10.79 \mathrm{~b}$ & $18.05 \pm 10.19 \mathrm{~b}$ \\
Filadelfia & $19.04 \pm 18.19 \mathrm{~b}$ & $47.75 .58 .96 \mathrm{~b}$ & $78.32 \pm 155.01 \mathrm{ab}$ \\
DSH (0.05) & 15.26 & 55.92 & 121.37 \\
\hline
\end{tabular}

Medias \pm DE (desviación estándar) con letras iguales en las columnas no son estadísticamente diferentes (Tukey $\leq 0.05)$. DSH: diferencia significativa honesta. 
campo e invernadero, identificar los posibles polinizadores, con el fin de estimular la polinización e incrementar la producción en estos materiales.

\section{CONCLUSIONES}

Bajo las condiciones de este estudio, el crecimiento y la producción de frutos de genotipos de tomate de cáscara silvestre mexicano es afectado por el potencial osmótico en la solución nutritiva. Los materiales evaluados responden positivamente al potencial osmótico intermedio de -0.036 MPa. El genotipo 9b es sobresaliente en número y peso de frutos, por lo que tiene potencial para usarse en sistemas protegidos con fines de comercialización.

\section{BIBLIOGRAFÍA}

Bustomi Rosadi R. A., M. Senge, D. Suhandy and A. Tusi (2014) The effect of EC levels of nutrient solution on the growth, yield, and quality of tomatoes (Solanum lycopersicum) under the hydroponic system. Journal of Agricultural Engineering and Biotechnology 2:7-12.

Castro-Brindis R., A. Galvis-Spínola, P. Sánchez-García, A. Peña-Lomelí, M. Sandoval-Villa y G. Alcantar-González (2004) Demanda de nitrógeno en tomate de cáscara (Physalis ixocarpa Brot.). Revista Chapingo Serie Horticultura 10:147-152.

Cruz C. E., M. Sandoval V., V. H. Volke H., A. Can C. y J. Sánchez E. (2012) Efecto de mezclas de sustratos y concentración de la solución nutritiva en el crecimiento y rendimiento de tomate. Revista Mexicana de Ciencias Agrícolas 3:1361-1373.

Cruz-Crespo E., A. Can-Chulim, R. Bugarín-Montoya, J. Pineda-Pineda, R. Flores-Canales, P. Juárez-López y G. Alejo-Santiago (2014) Concentración nutrimental foliar y crecimiento de chile serrano en función de la solución nutritiva y el sustrato. Revista Fitotecnia Mexicana 37:289-295.

Gastelum-Osorio D. A., M. Sandoval-Villa, C. Trejo-López y R. Castro-Brindis (2013) Fuerza iónica de la solución nutritiva y densidad de plantación sobre la producción y calidad de frutos de Physalis peruviana L. Revista Chapingo Serie Horticultura 19:197-210, https://doi.org/10.5154/r.rchsh.2012.01.002

Goykovic C. V. y G. Saavedra R. (2007) Algunos efectos de la salinidad en el cultivo del tomate y prácticas agronómicas de su manejo. IDESIA (Chile) 25:47-58, https://doi.org/10.4067/S071834292007000300006

López-Sandoval J. A., E. J. Morales-Rosales, H. Vibrans, E. Uscanga-Mortera, O. Vargas-Ponce y M. Martínez-y-Díaz-De-Salas (2018a) Cultivo de especies silvestres del género Physalis y su relación con la sinantropía. Tropical and Subtropical Agroecosystems 21:303315

López-Sandoval J. A., E. J. Morales-Rosales, H. Vibrans y E. J. Morales-Morales (2018b) Tasa de asimilación neta y rendimiento de Physalis bajo cultivo en dos localidades. Revista Fitotecnia Mexicana 41:187-197, https://doi.org/10.35196/rfm.2018.2.187-197

Marcelis L. F. M., E. Heuvelink, L. R. Baan Hofman-Eijer, J. Den Bakker and L. B. Xue (2004) Flower and fruit abortion in sweet pepper in relation to source and sink strength. Journal of Experimental Botany 55:2261-2268, https://doi.org/10.1093/jxb/erh245

Martínez F. E., J. Sarmiento, G. Fischer y F. Jiménez (2008) Efecto de la deficiencia de N, P, K, Ca, Mg y B en componentes de producción y calidad de la uchuva (Physalis peruviana L.). Agronomía Colombiana 26:389-398.

Miranda-Villagómez É., J. A. Carrillo-Salazar, M. N. Rodríguez-Mendoza, M. T. Colinas-León, M. Livera-Muñoz y E. A. Gaytán-Acuña (2014) Crecimiento y calidad del tallo floral de Freesia $\times$ hybrida en hidroponía. Revista Fitotecnia Mexicana 37:31-39.

Peña-Lomelí A., J. J. Ponce-Valerio, F. Sánchez-del-Castillo y N. MagañaLira (2014) Desempeño agronómico de variedades de tomate de cáscara en invernadero y campo abierto. Revista Fitotecnia Mexicana 37:381-391.

Ponce V. J. J., A. Peña-Lomelí, J. E. Rodríguez-Pérez, R. Mora-Aguilar, R. Castro-Brindis y N. Magaña L. (2012) Densidad y poda en tres variedades de tomate de cáscara (Physalis ixocarpa Brot. ex Horm.) cultivado en invernadero. Revista Chapingo Serie Horticultura 18:325-332, https://doi.org/10.5154/r rchsh.2010.08.028

Ribeiro da C. A., I. Katz, A. P. Sousa y R. A. Martínez U. (2015) Índice SPAD en el crecimiento y desarrollo de plantas de lisianthus en función de diferentes dosis de nitrógeno en ambiente protegido. IDESIA (Chile) 33:97-105, https://doi.org/10.4067/S071834292015000200012

Rojas-Velázquez A. N., L. A. Valdez-Aguilar, L. M. Ruiz-Posadas, M. SandovalVilla y V. Bertolini (2013) Respuestas de Antirrhinum majus (L.) para flor de corte al potencial osmótico de la solución nutritiva en dos estaciones de crecimiento. Revista Mexicana de Ciencias Agrícolas 4:1151-1160, https://doi.org/10.29312/ remexca.v4i8.1129

Ruiz E. F. H., R. L. Villalpando G., B. Murillo A., F. A. Beltrán M. y L. G. Hernández M. (2014) Respuesta diferencial a la salinidad de genotipos de tomate (Lycopersicon esculentum Mill.) en primeras etapas fenológicas. Terra Latinoamericana 32:311-323.

Sánchez-Díaz M. y J. Aguirreolea (2008) El agua en la planta. Movimiento del agua en el sistema suelo-planta-atmósfera. In: Fundamentos de Fisiología Vegetal. 2a edición J. Azcón-Bieto y M. Talón (eds.). McGraw-Hill Interamericana. Madrid, España. pp:25-39

SAS Institute (2011) SAS/STAT Version 9.3 User's Guide.. SAS Institute Inc. Cary, North Carolina, USA. 8621 p.

Steiner A. A. (1984) The universal nutrient solution. In: Proceedings of the $6^{\text {th }}$ International Congress on Soilless Culture. International Society for Soilless Culture. Wageningen, The Netherlands. pp: 633-649.

Taiz L. and E. Zeiger (2002) Plant Physiology. Third edition. Sinauer Associates. Sunderland, Massachusetts, USA. 690 p.

Urrieta-Velázquez J. A., M. N. Rodríguez-Mendoza, P. Ramírez-Vallejo, G. A Baca-Castillo, L. M. Ruiz-Posada y J. A. Cueto-Wong (2012) Variables de producción y calidad de tres selecciones de jitomate de costilla (Solanum lycopersicum L.). Revista Chapingo Serie Horticultura 18:371-381, https://doi.org/10.5154/r rchsh.2010.05.016

Valentín-Miguel M. C., R. Castro-Brindis, J. E. Rodríguez-Pérez y M. PérezGrajales (2013) Extracción de macronutrimentos en chile de agua (Capsicum annuum L.). Revista Chapingo Serie Horticultura 19:71-78, https://doi.org/10.5154/r.rchsh.2012.02.013

Valenzuela L. M., L. Partida R., T. Díaz V., T. J. Velázquez A., G. Bojórquez B. y T. Enciso 0. (2014) Respuesta del tomate cultivado en hidroponía con soluciones nutritivas en sustrato humus de lombriz-fibra de coco. Revista Mexicana de Ciencias Agrícolas 5:807-818, https://doi.org/10.29312/remexca.v5i5.903

Wubs A. M., Y. Ma, E. Heuvelink and L. F. M. Marcelis (2009) Genetic differences in fruit-set patterns are determined by differences in fruit sink strength and a source: sink threshold for fruit set. Annals of Botany 104:957-964, https://doi.org/10.1093/aob/ mcp181 
